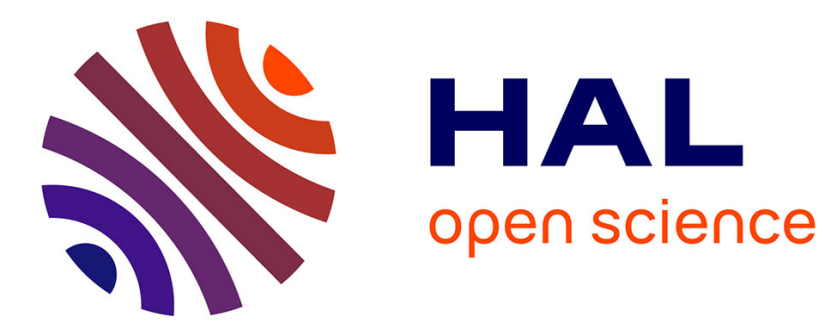

\title{
Asymptotic behavior of imaginary zeros of linear systems with commensurate delays
}

Jie Chen, Peilin Fu, Silviu-Iulian Niculescu

\section{To cite this version:}

Jie Chen, Peilin Fu, Silviu-Iulian Niculescu. Asymptotic behavior of imaginary zeros of linear systems with commensurate delays. 45th IEEE Conference on Decision and Control, Dec 2006, San Diego, CA, United States. 10.1109/CDC.2006.377014 . hal-02195825

\section{HAL Id: hal-02195825 \\ https://hal.science/hal-02195825}

Submitted on 26 Jul 2019

HAL is a multi-disciplinary open access archive for the deposit and dissemination of scientific research documents, whether they are published or not. The documents may come from teaching and research institutions in France or abroad, or from public or private research centers.
L'archive ouverte pluridisciplinaire HAL, est destinée au dépôt et à la diffusion de documents scientifiques de niveau recherche, publiés ou non, émanant des établissements d'enseignement et de recherche français ou étrangers, des laboratoires publics ou privés. 


\title{
Asymptotic Behavior of Imaginary Zeros of Linear Systems with Commensurate Delays
}

\author{
Jie Chen, Peilin Fu and Silviu-Iulian Niculescu
}

\begin{abstract}
This paper addresses the problem of asymptotic stability of linear time-delay systems with commensurate delays. We study the asymptotic behavior of the critical characteristic zeros of such systems on the imaginary axis. This behavior determines whether the imaginary zeros cross from one half plane into another, and hence plays a critical role in determining the stability of a time-delay system. We consider time-delay systems given in both state-space form and as a quasipolynomial. Our results reveal that in the former case the zero asymptotic behavior can be characterized by solving a simple eigenvalue problem, and in the latter case, by computing the derivatives of the quasipolynomial. To perform such an analysis, we make use of an operator perturbation approach.
\end{abstract}

Keywords: Time-delay, asymptotic stability, critical zeros, asymptotic behavior, matrix pencil.

\section{INTRODUCTION}

In this paper we consider linear time-delay systems described by the state-space equation

$$
\dot{x}(t)=A_{0} x(t)+\sum_{k=1}^{q} A_{k} x(t-k \tau), \quad \tau \geq 0 .
$$

Alternatively, we also consider the differential-difference equation

$$
y^{(n)}(t)+\sum_{i=0}^{n-1} \sum_{k=0}^{q} a_{k i} y^{(i)}(t-k \tau)=0, \quad \tau \geq 0 .
$$

It is known that both these representations provide full description of retarded delay systems with commensurate delays, which can be exchanged from one to another. We study the stability of these systems.

The stability of linear time-delay systems has been a wellstudied topic, which generally can be conducted equivalently on a system's characteristic quasipolynomial. For retarded systems with commensurate delays, the stability problem can be tackled in an especially efficient manner. Indeed, it is now widely known that the stability properties of such a system can be fully characterized by finding a set of critical delay values, at which the system's characteristic quasipolynomial has zeros on the imaginary axis. The latter can be computed in a variety of ways, notably, e.g., by solving a generalized

This research is supported in part by CNRS/France and NSF/USA.

J. Chen is with the department of Electrical Engineering, University of California, Riverside, CA92521, USA jchen@ee.ucr.edu

$\mathrm{P}$. $\mathrm{Fu}$ is with the Department of Electrical Engineering, University of California, Riverside, CA92521, USA pfu@ee. ucr.edu

S.I. Niculescu is with the HeuDiaSyC (UMR CNRS 6599), Centre de Recherche de Royallieu, Université de Technologie de Compiègne, BP 20529, 60205, Compiègne, France niculescu@hds.utc.fr eigenvalue problem [8]. These critical delay values form adjacent intervals; by the continuity of the zeros with respect to the delay, whether the system is stable or not over a specific delay interval can be determined by checking the stability for any one fixed value within that interval. Thus in this way, the stability of the system can be determined in principle over the entire range of delay values. One should note, nevertheless, that even for a fixed delay, the testing of stability for a time-delay system is not a simple task.

An alternative method in determining the stability is to analyze the asymptotic behavior of the critical zeros on the imaginary axis. Specifically, at each critical delay value corresponding to a critical zero of the characteristic quasipolynomial, we may seek to determine whether the zero may traverse from one half plane into another; for example, the system will become unstable if a critical zero enters the open right half plane, and otherwise will remain stable if all the critical zeros remain in the left half plane. This thinking has been advocated in the early works [2], [4], [5], [9], [13], [14], [16], and will be the central issue addressed in the present paper. This analysis of the zero asymptotic behavior, known as the stability switch problem, is unfortunately, not without intricacy. The issue is especially complicated by the fact that the characteristic zeros of a quasipolynomial may exhibit rather complex analytical properties, and as such a rigorous justification is called for; this seems to have been overlooked in the existing literature.

We adopt an operator perturbation approach to the stability switch problem. A sound mathematical tool (see, e.g., [11]), this approach appears most natural. Indeed, the approach seeks to recast the zero asymptotic analysis problem as one of eigenvalue perturbation. This enables us to obtain readily computable results that fully characterize the variation properties of the critical zeros at the critical delay values. Specifically, we show that the zero asymptotic behavior can be determined by computing the eigenvalues of a constant matrix, whereas the matrix in question is constructed directly from the solution of of the aforementioned generalized eigenvalue problem. As such, not only does the approach lend a rigorous treatment, but it also requires essentially no more computation than that required for determining the critical zeros. The results thus constitute a natural continuation of the previous work, and the computation overhead is minimal.

The remaining paper is organized as follows. In Section 2, we introduce and more importantly, develop further the eigenvalue perturbation results. These results are not only 
pertinent to our subsequent analysis, but in fact provide a more general development of interest to operator perturbation theory in general. Based on these results, we then solve in Section 3 the stability switch problem for the system given in (1). Section 4 develops companion results for systems described by (2), or equivalently, those described by a quasipolynomial. Section 5 concludes the paper.

The following notations will be used throughout the paper. Let $\mathbb{R}$ be the set of real numbers, $\mathbb{C}$ the set of complex numbers, and $\mathbb{R}_{+}$the set of nonnegative real numbers. Denote the open right half plane by $\mathbb{C}_{+}:=\{s: \Re(s)>0\}$, the closed right half plane by $\overline{\mathbb{C}}_{+}$, and the imaginary axis by $\partial \mathbb{C}_{+}$. Similarly, denote the open unit disc by $\mathbb{D}$, the unit circle by $\partial \mathbb{D}$, and the closed exterior of the unit disc by $\mathbb{D}^{c}$. For a matrix $A$, denote its spectrum by $\lambda(A)$, and the ith eigenvalue by $\lambda_{i}(A)$. For a matrix pair $(A, B)$, denote the set of all its generalized eigenvalues by $\sigma(A, B)$, i.e.,

$$
\sigma(A, B):=\{\lambda \in \mathbb{C}: \operatorname{det}(A-\lambda B)=0\} .
$$

The operation $A \oplus B$ denotes the Kronecker sum, and $A \otimes B$ the Kronecker product, of the matrices $A$ and $B$.

\section{Preliminary Results}

In this section, we introduce the operator perturbation theory for matrix eigenvalue problems, which concerns how the eigenvalues of a matrix function may vary with respect to a small perturbation. The development is based on the classical treatise of Kato [11], but goes further beyond.

Consider a matrix operator $T(x)$ of a real variable $x$. Suppose that in the neighborhood of $x=0$, the perturbed operator $T(x)$ is holomorphic, or equivalently, can be expanded into the power series,

$$
T(x)=T(0)+x T^{\prime}(0)+x^{2} T^{\prime \prime}(0)+\cdots .
$$

Let $D_{0}$ be a small disk near $x=0$ but excluding $x=0$. It is known that for $x \in D_{0}$, any semisimple eigenvalue of $T(x)$, namely the semisimple root of the characteristic equation

$$
\operatorname{det}(T(x)-\xi I)=0,
$$

is an analytic functions of $x$ and can also be expressed as a power series in $x$; here by a semisimple eigenvalue, we mean a repeated but diagonalizable eigenvalue. This fact is summarized in Lemma 1.

Lemma 1 [11] Let $\lambda^{(0)}$ be a semisimple eigenvalue of $T(0)$ with multiplicity $m$, and $P$ be the eigenprojection for $\lambda^{(0)}$, that is,

$$
P=\frac{1}{2 \pi j} \oint_{\Gamma}(\xi I-T(0))^{-1} d \xi
$$

where $\Gamma$ is a positively-oriented closed contour enclosing $\lambda^{(0)}$ but no other eigenvalues of T(0). Then the corresponding eigenvalues of $T(x)$ are analytic in $x$ and have the form

$$
\mu_{i}(x)=\lambda^{(0)}+\lambda_{i}^{(1)} x+o\left(x^{2}\right), \quad i=1, \ldots, m,
$$

where $\lambda_{i}^{(1)}$ are the eigenvalues of $P T^{\prime}(0) P$.
With no loss of generality, let $\lambda^{(0)}$ be ordered as the first eigenvalue of $T(0)$ with multiplicity $m$. Then $T(0)$ can be decomposed as

$$
T(0)=Q \Sigma R=\left[\begin{array}{ll}
Q_{1} & Q_{2}
\end{array}\right]\left[\begin{array}{cc}
\Sigma_{1} & 0 \\
0 & \Sigma_{2}
\end{array}\right]\left[\begin{array}{l}
R_{1} \\
R_{2}
\end{array}\right],
$$

where $\Sigma_{1}$ is diagonal with diagonal entries as $\lambda^{(0)}, R=$ $Q^{-1}=\left[\begin{array}{lll}r_{1}^{T} & \cdots & r_{n}^{T}\end{array}\right]^{T}$, and $Q=\left[\begin{array}{lll}q_{1} & \cdots & q_{n}\end{array}\right]$ consist of the eigenvectors and generalized eigenvectors of of $T(0)$. The following lemma shows how $\lambda_{i}^{(1)}$ may be computed.

Lemma 2 Let $T(0)$ be partitioned as in (7). Then $\lambda_{i}^{(1)}, i=$ $1, \ldots, m$, in (6) are the eigenvalues of $R_{1} T^{\prime}(0) Q_{1}$.

Proof. According to the Cauchy integral theorem,

$$
\begin{aligned}
P & =\frac{1}{2 \pi j} \oint_{\Gamma}(\xi I-T(0))^{-1} d \xi \\
& =Q\left[\frac{1}{2 \pi j} \oint_{\Gamma}(\xi I-\Sigma)^{-1} d \xi\right] R \\
& =Q\left[\begin{array}{ll}
I & 0 \\
0 & 0
\end{array}\right] R=Q_{1} R_{1} .
\end{aligned}
$$

As a result, we have

$$
\begin{aligned}
\lambda_{i}\left(P T^{\prime}(0) P\right) & =\lambda_{i}\left(Q_{1} R_{1} T^{\prime}(0) Q_{1} R_{1}\right) \\
& =\lambda_{i}\left(R_{1} T^{\prime}(0) Q_{1} R_{1} Q_{1}\right) \\
& =\lambda_{i}\left(R_{1} T^{\prime}(0) Q_{1}\right)
\end{aligned}
$$

where the last equality holds since $R_{1} Q_{1}=I$. This completes the proof.

Consider next the case that $\lambda^{(0)}$ is not a semisimple but repeated eigenvalue of $T(0)$ with multiplicity $\mathrm{m}$. In this case, $T(0)$ admits a Jordan decomposition in which $\Sigma$ is block diagonal with diagonal Jordan blocks, and $Q_{1}$ consists of the generalized eigenvectors associated with $\lambda^{(0)}$. In particular,

$$
\Sigma_{1}=\left[\begin{array}{cccc}
\lambda^{(0)} & 1 & \cdots & 0 \\
0 & \ddots & \ddots & 0 \\
\vdots & & \ddots & 1 \\
0 & \cdots & \cdots & \lambda^{(0)}
\end{array}\right] .
$$

The eigenvalue of $T(x)$ can no longer be expanded in the form of (6), but instead as a Puiseux series.

Lemma 3 Let $\lambda^{(0)}$ be a non-semisimple eigenvalue of $T(0)$ with multiplicity $m$. Then the corresponding eigenvalues of $T(x)$ have the form

$$
\mu_{i}(x)=\lambda^{(0)}+\left(\gamma_{i}^{(1)}\right)^{\frac{1}{m}} x^{\frac{1}{m}}+\cdots, \quad i=1, \ldots, m,
$$

where $\gamma_{i}^{(1)}=r_{m} T^{\prime}(0) q_{1}$.

Proof. Construct an operator $W(x)$ as

$$
W(x)=\left(T(x)-\lambda^{(0)} I\right)^{m} .
$$


It follows that

$$
\begin{aligned}
W(0) & =\left(T(0)-\lambda^{(0)} I\right)^{m}=\left(Q \Sigma R-\lambda^{(0)} I\right)^{m} \\
& =Q\left(\Sigma-\lambda^{(0)} I\right)^{m} R .
\end{aligned}
$$

As a result, 0 is a semisimple eigenvalue of $W(0)$ with multiplicity $m$. Since $W(x)$ is holomorphic, i.e.,

$$
W(x)=W(0)+x W^{\prime}(0)+\cdots,
$$

Lemma 1 indicates that the eigenvalues of $W(x)$ near $x=0$ can be expanded as

$$
\nu_{i}(x)=0+\gamma_{i}^{(1)} x+o\left(x^{2}\right), \quad i=1, \ldots, m
$$

where $\gamma_{i}^{(1)}$ are the eigenvalues of $P_{W} W^{\prime}(0) P_{W}$, with

$$
P_{W}=\frac{1}{2 \pi j} \oint_{\Gamma}(\xi I-W(0))^{-1} d \xi,
$$

and $\Gamma$ as a positively-oriented closed contour enclosing 0 but no other eigenvalues of $W(0)$. On the other hand, according to (9), the eigenvalues of $W(x)$ are found to be

$$
\begin{aligned}
\lambda_{i}(W(x)) & =\lambda_{i}\left(\left(T(x)-\lambda^{(0)} I\right)^{m}\right) \\
& =\left[\lambda_{i}(T(x))-\lambda^{(0)}\right]^{m} .
\end{aligned}
$$

As a result, the $i$-th eigenvalue of $T(x)$, namely $\mu_{i}(x)$, satisfies

$$
\left[\mu_{i}(x)-\lambda^{(0)}\right]^{m}=0+\gamma_{i}^{(1)} x+\cdots, \quad i=1,2, \cdots, m,
$$

which gives rise to

$$
\mu_{i}(x)=\lambda^{(0)}+\left(\gamma_{i}^{(1)}\right)^{\frac{1}{m}} x^{\frac{1}{m}}+\cdots .
$$

The remainder of the proof then amounts to calculating $\gamma_{i}^{(1)}, i=1,2, \cdots, m$, which are the eigenvalues of $P_{W} W^{\prime}(0) P_{W}$. Since

$$
\begin{aligned}
P_{W} & =\frac{1}{2 \pi j} \oint_{\Gamma}(\xi I-W(0))^{-1} d \xi \\
& =\frac{1}{2 \pi j} \oint_{\Gamma} Q\left[\xi I-\left(\Sigma-\lambda^{(0)} I\right)^{m}\right]^{-1} R d \xi \\
& =Q\left[\begin{array}{ll}
I & 0 \\
0 & 0
\end{array}\right] R \\
& =Q_{1} R_{1},
\end{aligned}
$$

we have $\lambda_{i}\left(P_{W} W^{\prime}(0) P_{W}\right)=\lambda_{i}\left(Q_{1} R_{1} W^{\prime}(0) Q_{1} R_{1}\right)=$ $\lambda_{i}\left(R_{1} W^{\prime}(0) Q_{1}\right)$, where $Q_{1}=\left[\begin{array}{llll}q_{1} & q_{2} & \cdots & q_{m}\end{array}\right]$ and $R_{1}=$ $\left[\begin{array}{llll}r_{1}^{T} & r_{2}^{T} & \cdots & r_{n}^{T}\end{array}\right]^{T}$. Since $q_{1}, q_{2}, \cdots, q_{m}$ are the generalized eigenvectors associated with $\lambda^{(0)}$, it follows that

$$
\begin{aligned}
{\left[T(0)-\lambda^{(0)} I\right] q_{1} } & =\left[T(0)-\lambda^{(0)} I\right]^{2} q_{2} \\
& =\cdots \\
& =\left[T(0)-\lambda^{(0)} I\right]^{m} q_{m} \\
& =0 .
\end{aligned}
$$

Furthermore, consider $W(x)$ as the $m$ successive products of $T(x)-\lambda^{(0)} I$, we have

$W^{\prime}(0)=\sum_{k=0}^{m-1}\left[T(0)-\lambda^{(0)} I\right]^{k} T^{\prime}(0)\left[T(0)-\lambda^{(0)} I\right]^{m-1-k}$.

It then follows from (11) and (12) that

$$
\begin{aligned}
W^{\prime}(0) q_{1} & =\left[T(0)-\lambda^{(0)} I\right]^{m-1} T^{\prime}(0) q_{1} \\
W^{\prime}(0) q_{2} & =\left[T(0)-\lambda^{(0)} I\right]^{m-2} T^{\prime}(0)\left[T(0)-\lambda^{(0)} I\right] q_{2} \\
& +\left[T(0)-\lambda^{(0)} I\right]^{m-1} T^{\prime}(0) q_{2} \\
& =\left[T(0)-\lambda^{(0)} I\right]^{m-2} T^{\prime}(0) q_{1} \\
& +\left[T(0)-\lambda^{(0)} I\right]^{m-1} T^{\prime}(0) q_{2} \\
& \vdots \\
W^{\prime}(0) q_{m} & =\left[T(0)-\lambda^{(0)} I\right]^{0} T^{\prime}(0) q_{1} \\
& +\left[T(0)-\lambda^{(0)} I\right]^{1} T^{\prime}(0) q_{2}+\cdots \\
& +\left[T(0)-\lambda^{(0)} I\right]^{m-1} T^{\prime}(0) q_{m}
\end{aligned}
$$

Moreover, the Jordan decomposition $T(0)=Q \Sigma R$ leads to

$$
\left[T(0)-\lambda^{(0)} I\right]^{k}=Q\left[\Sigma-\lambda^{(0)} I\right]^{k} R \text {. }
$$

As a consequence, we have

$$
\begin{aligned}
r_{1}[T(0) & \left.-\lambda^{(0)} I\right]^{k} \\
= & r_{1} Q\left[\Sigma-\lambda^{(0)} I\right]^{k} R \\
= & {\left[\begin{array}{llll}
1 & 0 & \cdots & 0
\end{array}\right]\left[\Sigma-\lambda^{(0)} I\right]^{k} R } \\
= & \left\{\begin{array}{cc}
r_{2} & k=1 \\
r_{3} & k=2 \\
\vdots & \vdots \\
r_{m} & k=m-1
\end{array}\right. \\
r_{2}[T(0) & \left.-\lambda^{(0)} I\right]^{k} \\
= & {\left[\begin{array}{ccc}
0 & 0 & \cdots
\end{array}\right]\left[\Sigma-\lambda^{(0)} I\right]^{k} R } \\
= & \left\{\begin{array}{cc}
r_{3} & k=1 \\
r_{4} & k=2 \\
\vdots & \vdots \\
r_{m} & k=m-2 \\
0 & k=m-1
\end{array}\right.
\end{aligned}
$$

In light of (13) and (16), it is then straightforward to verify that

$r_{1} W^{\prime}(0) q_{1}=r_{1}\left[T(0)-\lambda^{(0)} I\right]^{m-1} T^{\prime}(0) q_{1}=r_{m} T^{\prime}(0) q_{1}$. 
Similarly,

$$
r_{i} W^{\prime}(0) q_{j}= \begin{cases}r_{m} T^{\prime}(0) q_{1} & i=j \\ 0 & i>j\end{cases}
$$

Consequently,

$$
\begin{aligned}
& R_{1} W^{\prime}(0) Q_{1} \\
& =\left[\begin{array}{cccc}
r_{m} T^{\prime}(0) q_{1} & r_{1} W^{\prime}(0) q_{2} & \cdots & r_{1} W^{\prime}(0) q_{m} \\
0 & r_{m} T^{\prime}(0) q_{1} & \cdots & r_{2} W^{\prime}(0) q_{m} \\
\vdots & \vdots & \ddots & \vdots \\
0 & 0 & \cdots & r_{m} T^{\prime}(0) q_{1}
\end{array}\right]
\end{aligned}
$$

which is an upper triangular matrix with the eigenvalues

$$
\gamma_{i}^{(0)}=r_{m} T^{\prime}(0) q_{1}, i=1,2, \cdots m .
$$

This completes the proof.

It is worth pointing out that most of the available literature (see, e.g., [?], [15], [12]), while noting that $\mu_{i}(x)$ can be expanded in a Puiseux series, does not provide a characterization of the coefficients, which, however, will be of central importance in our subsequent development. On the other hand, the explicit expression (8) has been given in [1] (pp. 164) without proof, and derived in [6]; the derivation in the latter reference, unfortunately, was done in a seemingly ad hoc way. Our development gives a rigorous treatment of this case based on the semisimple case developed in [11], which also appears more elegant mathematically.

\section{State-Space Models}

We now consider the systems described by the state-space form (1). The characteristic quasipolynomial associated with this system is given by

$$
p(s, \tau):=\operatorname{det}\left(s I_{n}-\sum_{k=0}^{q} A_{k} e^{-s k \tau}\right) .
$$

For a fixed $\tau \geq 0$, the system is asymptotically stable if and only if all the zeros of the quasipolynomial $p(s, \tau)$ lie in $\mathbb{C}^{-}$(see, e.g., [8]). Define the matrices

$$
\begin{aligned}
U= & {\left[\begin{array}{ccccc}
I_{n^{2}} & 0 & \ldots & 0 & 0 \\
0 & I_{n^{2}} & \ldots & 0 & 0 \\
& & \ddots & & \\
0 & 0 & \ldots & I_{n^{2}} & 0 \\
0 & 0 & \ldots & 0 & B_{2 q}
\end{array}\right], } \\
V= & {\left[\begin{array}{ccccc}
0 & I_{n^{2}} & 0 & \ldots & 0 \\
0 & 0 & I_{n^{2}} & \ldots & 0 \\
& & & \ddots & \\
0 & 0 & 0 & \ldots & I_{n^{2}} \\
-B_{0} & -B_{1} & -B_{2} & \ldots & -B_{2 q-1}
\end{array}\right] }
\end{aligned}
$$

where $B_{m} \in \mathbb{R}^{n^{2}}, m=0,1, \ldots, 2 q$ are given by

$$
B_{q-m}=I_{n} \otimes A_{m}^{T}, \quad B_{q}=A_{0} \oplus A_{0}^{T}, \quad B_{q+m}=A_{m} \otimes I_{n} .
$$

The following Lemma characterizes the critical zeros of $p(s, \tau)$ on the imaginary axis, together with the critical delay values (see, e.g., [3]).
Lemma 4 The quasipolynomial $p(s, \tau)$ has a critical zero on the imaginary axis if and only if the following conditions are satisfied:

(i) $\sigma(V, U) \cap \partial \mathbb{D} \neq \emptyset$;

(ii) There exists some $z_{i} \in \sigma(V, U) \cap \partial \mathbb{D}$ such that

$$
\sigma\left(\sum_{k=0}^{q} A_{k} z_{i}^{k}\right) \cap j \mathbb{R}_{+} \neq \emptyset .
$$

For a critical zero $j \omega_{i} \in \sigma\left(\sum_{k=0}^{q} A_{k} z_{i}^{k}\right)$ where $\omega_{i} \in \mathbb{R}_{+}$, $\omega_{i} \neq 0$, the corresponding critical delay is an element from the set

$$
\mathcal{T}\left(\omega_{i}\right)=\left\{\frac{\log \left(\overline{z_{i}}\right)}{j \omega_{i}}+\frac{2 \pi \ell}{\omega_{i}}>0, \ell=1,2, \ldots\right\},
$$

where $\log (\cdot)$ represents the Cauchy principal value.

Evidently, since the zeros of $p(s, \tau)$ are conjugate symmetric, it suffices to consider only the critical zeros with $\omega_{i}>0$. This is reflected in the lemma.

Let $\tau^{*}$ and $j \omega^{*}, \omega^{*} \in \mathbb{R}_{+}, \omega^{*} \neq 0$ be a critical pair of critical delay and critical zero of $p(s, \tau)$, i.e., $p\left(j \omega^{*}, \tau^{*}\right)=$ 0 . Introduce the new real variable $x=\tau-\tau^{*}$, and define

$$
T(x):=\sum_{k=0}^{q}\left(A_{k} e^{-j \omega^{*} k \tau^{*}}\right) e^{-j \omega^{*} k x} .
$$

Clearly, $T(x)$ is holomorphic. Furthermore, since

$$
\operatorname{det}\left(T(0)-j \omega^{*} I\right)=p\left(j \omega^{*}, \tau^{*}\right)=0,
$$

$j \omega^{*}$ is an eigenvalue of $T(0)$. Without loss of generality, let $j \omega^{*}$ be ordered as the first eigenvalue of $T(0)$, with multiplicity $m$. We first consider the case that $j \omega^{*}$ is a semisimple eigenvalue.

Theorem 1 Let $j \omega^{*}$ be a semisimple eigenvalue of $\sum_{k=0}^{q} A_{k} e^{-j \omega^{*} k \tau^{*}}$. Then for any $\tau$ sufficiently close to $\tau^{*}$, the characteristic zeros corresponding to $j \omega^{*}$ can be expanded by the power series

$$
\begin{gathered}
j \omega^{*}-\lambda_{i}\left[R_{1}\left(\sum_{k=1}^{q} j k \omega^{*} A_{k} e^{-j \omega^{*} k \tau^{*}}\right) Q_{1}\right]\left(\tau-\tau^{*}\right) \\
+o\left(\left(\tau-\tau^{*}\right)^{2}\right), \quad i=1,2, \cdots, m .
\end{gathered}
$$

Thus, for $\tau$ sufficiently close to $\tau^{*}$ but $\tau>\tau^{*}$, there are at least $\ell(\ell \leq m)$ of characteristic zeros entering the righthalf plane (or vice versa) if $\ell$ of the eigenvalues satisfy the condition

$$
\operatorname{Re}\left\{\lambda_{i}\left[R_{1}\left(\sum_{k=1}^{q} j k \omega^{*} A_{k} e^{-j \omega^{*} k \tau^{*}}\right) Q_{1}\right]\right\}<0(>0)
$$$$
i=1,2, \cdots, m(21)
$$

where $Q_{1}=\left[\begin{array}{llll}q_{1} & q_{2} & \cdots & q_{m}\end{array}\right]$ and $R_{1}=\left[\begin{array}{llll}r_{1}^{T} & r_{2}^{T} & \cdots & r_{m}^{T}\end{array}\right]^{T}$, with $q_{i}$ and $r_{i}$ the right and left eigenvectors of $\sum_{k=0}^{q} A_{k} e^{-j \omega^{*} k \tau^{*}}$ associated with $j \omega^{*}$. 
Proof. The result follows from a direct application of Lemma 2, by noting that near $x=0$ (i.e., near $\tau=\tau^{*}$ ), the matrix function given in (19) can be expanded as

$$
\begin{aligned}
T(x) & =T(0)+x T^{\prime}(0)+o\left(x^{2}\right) \\
& =\sum_{k=0}^{q} A_{k} e^{-j \omega^{*} k \tau^{*}}-x\left(\sum_{k=1}^{q} j k \omega^{*} A_{k} e^{-j \omega^{*} k \tau^{*}}\right) \\
& +o\left(x^{2}\right) .
\end{aligned}
$$

This gives the power series expansion (20). Taking the real part of (20) then completes the proof.

It is worth noting that the right and left eigenvectors in Theorem 1 are precisely those of the matrix $\sum_{k=0}^{q} A_{k} z_{i}^{k}$, with $z_{i}=e^{-j \omega^{*} \tau^{*}}$. Hence, the matrices $Q_{1}$ and $R_{1}$ are computed directly while computing the spectrum of $\sum_{k=0}^{q} A_{k} z_{i}^{k}$, which is required in order to determine the critical zeros $j \omega^{*}$. The computation required to check the condition (21) is then only that of computing the eigenvalues. In particular, when $j \omega^{*}$ is a simple critical zero, this computation can be further simplified. The following corollary is an immediate consequence of Theorem 1, which attests to this observation.

Corollary 1 Let $j \omega^{*}$ be a simple eigenvalue of $\sum_{k=0}^{q} A_{k} e^{-j \omega^{*} k \tau^{*}}$. Then for any $\tau$ sufficiently close to $\tau^{*}$ but $\tau>\tau^{*}, j \omega^{*}$ enters the right-half plane (or vice versa) if

$$
\operatorname{Re}\left\{r_{1}\left(\sum_{k=1}^{q} j k \omega^{*} A_{k} e^{-j \omega^{*} k \tau^{*}}\right) q_{1}\right\}<0 \quad(>0),
$$

where $q_{1}$ and $r_{1}$ are the right and left eigenvectors associated with $j \omega^{*}$.

Our next result concerns the case that $j \omega^{*}$ is not a semisimple but repeated eigenvalue. The proof is analogous to that of Theorem 1, and follows from Lemma 3.

Theorem 2 Let $j \omega^{*}$ be a repeated eigenvalue of $\sum_{k=0}^{q} A_{k} e^{-j \omega^{*} k \tau^{*}}$ with multiplicity $m$. Suppose that $j \omega^{*}$ is not semisimple. Then for any $\tau$ sufficiently close to $\tau^{*}$ but $\tau>\tau^{*}$, the characteristic zeros corresponding to $j \omega^{*}$ can be expanded by the Puiseux series

$$
\begin{aligned}
j \omega^{*} & +\left|r_{m}\left(\sum_{k=1}^{q} j k \omega^{*} A_{k} e^{-j \omega^{*} k \tau^{*}}\right) q_{1}\right|^{\frac{1}{m}} e^{j \frac{2 k \pi+\pi+\theta}{m}} \\
& \times\left(\tau-\tau^{*}\right)^{\frac{1}{m}}+\cdots, \quad k=0,1, \cdots, m-1,
\end{aligned}
$$

where $\theta \in[0,2 \pi]$ is the phase angle of $r_{m}\left(\sum_{k=1}^{q} j k \omega^{*} A_{k} e^{-j \omega^{*} k \tau^{*}}\right) q_{1}$, and $q_{1}$ and $r_{m}$ are obtained from the Jordan decomposition

$$
\sum_{k=0}^{q} A_{k} e^{-j \omega^{*} k \tau^{*}}=Q \Sigma R
$$

with $Q=\left[\begin{array}{llll}q_{1} & q_{2} & \cdots & q_{n}\end{array}\right]$ and $R=Q^{-1}=\left[\begin{array}{llll}r_{1}^{T} & r_{2}^{T} & \cdots & r_{n}^{T}\end{array}\right]^{T}$. Hence, for $\tau$ sufficiently close to $\tau^{*}$ but $\tau>\tau^{*}$, the number of critical zeros entering the right-half plane (or vice versa) can be determined by the condition

$$
\cos \left(\frac{2 k \pi+\pi+\theta}{m}\right)>0 \quad(<0), \quad k=0, \cdots, m-1 .
$$

Theorem 2 reveals a fundamental difference between the asymptotic behaviors of a semisimple eigenvalue and one that is not. For a repeated eigenvalue that is not semisimple, the result shows that, precluding the case that

$$
r_{m}\left(\sum_{k=1}^{q} j k \omega^{*} A_{k} e^{-j \omega^{*} k \tau^{*}}\right) q_{1}=0,
$$

whether the zero will enter the right-half plane is solely determined by the multiplicity of the zero and the phase angle $\theta$. In this case, the branches of the zero will almost generically enter the right-half plane. Note that in the degenerate case $m=1$, Theorem 2 also reduces to Corollary 1 .

\section{Differential-Difference Equation Model}

In this section we develop companion results for systems described by the differential-difference equation (2), or equivalently, by the quasipolynomial

$$
a\left(s, e^{-\tau s}\right)=\sum_{k=0}^{q} a_{k}(s) e^{-k \tau s}, \quad \tau \geq 0,
$$

where

$a_{0}(s)=s^{n}+\sum_{i=0}^{n-1} a_{0 i} s^{i}, \quad a_{k}(s)=\sum_{i=0}^{n-1} a_{k i} s^{i}, \quad k=1, \cdots, q$.

We note that a similar generalized eigenvalue-based solution (see, e.g., [3]) exists for the critical zeros $j \omega^{*}$ of $a\left(s, e^{-\tau s}\right)$ at the critical delay value $\tau^{*}$, at which $a\left(j \omega^{*}, e^{-j \omega^{*} \tau^{*}}\right)=$ 0 . It is also clear that Theorem 1 and Theorem 2 can be directly applied to determine the asymptotic behavior of the critical zeros $j \omega^{*}$, by making use of the realization

$$
\begin{gathered}
A_{0}=\left[\begin{array}{cccc}
0 & 1 & \cdots & 0 \\
\vdots & \vdots & \ddots & \vdots \\
0 & 0 & \cdots & 1 \\
-a_{00} & -a_{01} & \cdots & -a_{0, n-1}
\end{array}\right], \\
A_{k}=\left[\begin{array}{cccc}
0 & 0 & \cdots & 0 \\
\vdots & \vdots & \ddots & \vdots \\
0 & 0 & \cdots & 0 \\
-a_{k 0} & -a_{k 1} & \cdots & -a_{k, n-1}
\end{array}\right], \quad k=1, \ldots, q .
\end{gathered}
$$

Additionally, with the specific structures of this realization, it is not difficult to derive that the eigenvalues of $T(0)$ are also the roots of quasipolynomial $a\left(s, e^{-j \omega^{*} \tau^{*}}\right)$ which make it possible to state the results directly in terms of $a\left(s, e^{-\tau s}\right)$. We first show that with a realization given by the above companion form, $T(0)$ cannot have semisimple repeated eigenvalues.

\section{Lemma 4 Any companion matrix}

$$
A=\left[\begin{array}{cccc}
0 & 1 & \cdots & 0 \\
\vdots & \vdots & \ddots & \vdots \\
0 & 0 & \cdots & 1 \\
-\alpha_{0} & -\alpha_{1} & \cdots & -\alpha_{n-1}
\end{array}\right]
$$


cannot have semisimple repeated eigenvalues.

Proof. Assume otherwise; that is, $A$ has a semisimple repeated eigenvalue $\lambda^{(0)}$ with multiplicity $m, m>1$. Then, there exists a similarity transformation such that $P A P^{-1}$ is in block diagonal form, in which, without loss of generality, the first diagonal block is itself an $m \times m$ diagonal matrix with diagonal elements equal to $\lambda^{(0)}$. Consider the vector $b=\left[\begin{array}{llll}0 & 0 & \cdots & 1\end{array}\right]^{T}$. It is well-known that $(A, b)$ forms a controllable pair. Since controllability is invariant under similarity transformation, $\left(P A P^{-1}, P b\right)$ is also controllable. This, however, is not possible with $P A P^{-1}$ given as above, a fact readily verified using, e.g., the $\mathrm{PBH}$ controllability test [10]. We are thus led to contradiction and the proof is completed.

The implication of Lemma 4 is rather clear. With the above realization of $A_{k}$, the matrix $T(0)$ is in the companion form with $\alpha_{i}=\sum_{k=0}^{q} a_{k i} e^{-j k \omega^{*} \tau^{*}}, i=0,1 \cdots, n-1$. As such, when applying the results in Section 3, one can preclude the case that $T(0)$ has semisimple repeated eigenvalues; instead, the critical zero $j \omega^{*}$ is either a simple eigenvalue, or a repeated, non-semisimple eigenvalue of $T(0)$. Theorem 3 and Corollary 2 given below describe the asymptotic behavior of imaginary poles in these two cases, in terms of $a\left(s, e^{-\tau s}\right)$. The proofs of these results involve lengthy algebraic manipulations and are omitted for brevity.

Theorem 3 Let $j \omega^{*}$ be a repeated zero of a $\left(s, e^{-j \omega^{*} \tau^{*}}\right)$ with multiplicity $m$. Then for any $\tau$ sufficiently close to $\tau^{*}$ but $\tau>\tau^{*}$, the zeros corresponding to $j \omega^{*}$ can be expanded by the Puiseux series

$$
\begin{array}{r}
j \omega^{*}+\left|m ! \frac{\left.\frac{d a\left(j \omega^{*}, e^{-j \omega^{*} \tau}\right)}{d \tau}\right|_{\tau=\tau^{*}}}{\left.\frac{d^{m} a\left(s, e^{-j \omega^{*} \tau^{*}}\right)}{d s^{m}}\right|_{s=j \omega^{*}}}\right|^{\frac{1}{m}} e^{j \frac{2 k \pi+\pi+\theta}{m}}\left(\tau-\tau^{*}\right)^{\frac{1}{m}} \\
+\cdots, \quad k=0,1, \cdots, m-1,
\end{array}
$$

where $\theta \in[0,2 \pi]$ is the phase angle of

$$
\frac{\left.\frac{d a\left(j \omega^{*}, e^{-j \omega^{*} \tau}\right)}{d \tau}\right|_{\tau=\tau^{*}}}{\left.\frac{d^{m} a\left(s, e^{-j \omega^{*} \tau^{*}}\right)}{d s^{m}}\right|_{s=j \omega^{*}}} .
$$

Hence, for $\tau$ sufficiently close to $\tau^{*}$ but $\tau>\tau^{*}$, the number of critical zeros entering the right-half plane (or vice versa) can be determined by the condition

$$
\cos \left(\frac{2 k \pi+\pi+\theta}{m}\right)>0 \quad(<0), \quad k=0,1, \cdots, m-1 .
$$

Corollary 2 Let $j \omega^{*}$ be a simple zero of a $\left(s, e^{-j \omega^{*} \tau^{*}}\right)$. Then for any $\tau$ sufficiently close to $\tau^{*}$ but $\tau>\tau^{*}, j \omega^{*}$ enters the right-half plane (or vice versa) if

$$
\operatorname{Re}\left\{\frac{\left.\frac{d a\left(j \omega^{*}, e^{-j \omega^{*} \tau}\right)}{d \tau}\right|_{\tau=\tau^{*}}}{\left.\frac{d a\left(s, e^{-j \omega^{*} \tau^{*}}\right)}{d s}\right|_{s=j \omega^{*}}}\right\}<0 \quad(>0) .
$$

It is useful to note that in the case of a simple critical zero, results similar to Corollary 3 have been reported in [2],
[4], [5], [9], [16], while without much technical deliberation. Our derivation herein thus provides an independent, rigorous justification to some of the previously available results.

\section{CONCLUDING REMARKS}

In this paper we have studied the asymptotic behavior of the critical characteristic zeros of linear time-delay systems with commensurate delays, for systems posed both in statespace form and as a quasipolynomial. The results build on the previous work on computing the critical delay value and critical zeros, and hence require no further significant computation.

While this paper is only concerned with retarded timedelay systems, the results can be extended in a fairly straightforward manner to neutral systems, based on the recent work [7] by the authors. It is also possible to carry out a more elaborate development utilizing second or even higher order analysis, to have a more refined characterization of the zero asymptotic behavior. This will require a series expansion in higher order terms. A second order analysis will be useful when the present first-order expansion ceases to be conclusive, when some of the eigenvalues in the firstorder term have a real part equal to zero. These extensions will be reported in a forthcoming paper.

\section{REFERENCES}

[1] H. Baumgärtel, Analytic Perturbation Theory for Matrices and Operators, Birkhäuser, Boston; 1985.

[2] F.G. Boese, Stability with respect to the delay: On the paper of K.L. Cooke and P. van den Driessche, J. Math. Anal.Appl., vol. 228, 1998, pp 293-321.

[3] J. Chen, C. Gu and C.N. Nett, A new method for computing delay margins for stability of linear delay systems, Syst. \& Contr. Lett., vol. 26, 1995, pp 101-117.

[4] K.L. Cooke and Z. Grossman, Discrete delay, distributed delay and stability switches, J. Math. Anal. Appl., vol. 86, 1982, pp 592-627.

[5] K.L. Cooke and P. Van den Driessche, On zeroes of some transcendental equations, Funkcialaj Ekvacioj, vol. 29, 1986, pp 77-90.

[6] A.S. Deif, Advanced Matrix Theory for Scientists and Engineers, Abacus Press, England; 1982.

[7] P. Fu, S.I. Niculescu, and J. Chen, "Stability of linear neutral timedelay systems: exact conditions via matrix pencil solutions", in Proceedings of 2005 American Control Conference, Portland, OR, 2005 pp 4259-4264.

[8] K. Gu, V.L. Kharitnov and J. Chen, Stability of Time-Delay Systems, Birkhäuser, Boston; 2003.

[9] D. Hertz, E.I. Jury and E. Zeheb, Simplified analytical stability test for systems with commensurate time delays, IEE Proc. Contr. Theory \& Appl., Pt. D, 131, 1984, pp 52-56.

[10] T. Kailath, Linear Systems, Prentice-Hall, Englewood Cliffs, NJ; 1980.

[11] T. Kato, Perturbation Theory for Linear Operations, Springer-Verlag, Berlin; 1995.

[12] P. Lancaster, A.S. Markus and F. Zhou, Perturbation theory for analytic matrix functions: the semisimple case, SIAM J. Matrix Anal. Appl., vol. 25 , no. 3, 2003, pp 606-626.

[13] M.S. Lee and C.S. Hsu, On the $\tau$-decomposition method of stability analysis for retarded dynamic systems, SIAM J. Control, vol. 7, no. 2, 1969, pp 242-259.

[14] N. Olgac, and R. Sipahi, An exact method for the stability analysis of time-delayed LTI systems, IEEE Trans. Automat. Contr., vol. 47, 2002, pp 793-797.

[15] J. Sun, Multiple eigenvalue sensitivity analysis, Linear Algebra and Its Applications, vol. 137/138, 1990, pp 183-211.

[16] K. Walton and J.E. Marshall, Direct Method for TDS Stability Analysis, IEE Proc. Contr. Theory \& Appl., 134, Pt. D:2, 1987, pp 101-107. 